\title{
Rassegna
}

\section{La nefropatia da contrasto. Definizione, fattori di rischio, prevenzione}

\author{
C. Guastoni, B. Gidaro, P. Covella \\ U.O. Nefrologia, A.O. Ospedale Civile di Legnano, Legnano
}

CONTRAST NEPHROPATHY. DEFINITION, RISK FACTORS, PROPHYLAXIS

ABstract. Contrast induced nephropathy (CIN) is one of the most frequent cause of acute kidney injury in recovered patients.

CIN incidence depends on patient's risk factors (i.e. chronic kidney disease, diabetes, cardiovascular diseases and older age) and procedure's related factors (i.e. high contrast dose, intra arterial administration). Chronic kidney disease, especially if associated with diabetes, is the main risk factor of CIN. Hydration before and after contrast administration is the only preventive therapy strongly recommended in guidelines in patients at risk for CIN.

CIN prevention studies have focused mainly on cardiological patients with moderate renal risk (GFR 60-40 $\mathrm{mL} / \mathrm{min}$ ) who underwent intra arterial contrast administration.

Many clinical trials have evaluated efficacy of hydration associated with sodium bicarbonate and of N acetylcysteine (NAC) in CIN prevention.

Sodium bicarbonate infusion has demonstrated better efficacy than saline infusion, particularly when short infusion times are needed, such as in emergency procedures.

NAC hasn't shown clear effect, in fact some positive studies results haven't been confirmed in other trials yet.

The discussion is still opened on the efficacy of renal replacement therapies in CIN prevention in high renal risk subjects $(\mathrm{GFR}<30 \mathrm{~mL} / \mathrm{min})$ in which $\mathrm{CIN}$ could associate to beginning of chronic dialysis.

KeY WorDS. Contrast, N-acetylcysteine, AKI

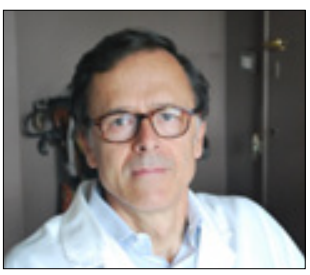

Carlo Guastoni

\section{Definizione}

Nella maggior parte dei trial clinici e nelle più recenti linee guida della Società Europea di Radiologia (ESUR) la nefropatia da mezzo di contrasto (MDC) (contrast induced nephropathy) (CIN) viene definita dall'aumento della creatininemia (valore assoluto $\geq 0.5 \mathrm{mg} / \mathrm{dL}$ o relativo $\geq 25 \%$ ) rispetto al basale dopo 48-72 ore dall'utilizzo del mezzo di contrasto, non correlabile ad altre cause $(1,2)$.

La definizione di CIN basata sulla creatinina ha, però, dei limiti perché considera solo l'effetto sulla funzione renale, mentre sarebbe auspicabile poter utilizzare dei markers di danno cellulare renale equivalente alle troponine in campo cardiologico.

Sono attualmente in fase di studio sia nel plasma $(\mathrm{N}$ GAL, cistatina C), che nelle urine (NGAL, KIM-1, IL-18, NAG) dei biomarkers di acute kidney iniury (AKI) più precoci e sensibili rispetto all'incremento della creatinina $(3,4)$ che sono stati valutati anche nella nefropatia da $\operatorname{MDC}(5,6)$.

\section{Fattori di rischio e incidenza di CIN}

Gli studi osservazionali su pazienti sottoposti a procedure cardiologiche con MDC intra-arterioso $(7,8)$ hanno evidenziato le condizioni cliniche (rischi legati al paziente) che comportano un'aumentata incidenza di CIN: insufficienza renale preesistente, diabete, età avanzata, scompenso cardiaco, ipovolemia, infarto miocardico, anemia, vasculopatia periferica, ipertensione arteriosa. Inoltre sono stati evidenziati come fattori di rischio legati alla procedura la presenza di ipotensione pre- ed intraprocedura e l'utilizzo del contropulsatore.

La più grave condizione clinica di rischio di CIN è senza dubbio rappresentata dalla riduzione della funzione renale. L'incidenza di CIN è pressoché assente nei pazienti con funzione renale normale e varia dal 5 al $20 \%$ 
nelle diverse casistiche (9) di pazienti con insufficienza renale moderata.

Nei pazienti con diabete ed insufficienza renale preesistente può raggiungere anche il $50 \%$ dopo procedur-e coronariche (PCI) (10).

La misura del filtrato renale (GFR) ottenuta con la formula MDRD $(11,12)$ o con la formula CKD-EPI (13) rappresenta il marker della funzione renale da utilizzare per lo screening dei pazienti a rischio.

Il valore soglia (cut off) del GFR nei confronti del rischio di CIN nella somministrazione intra-arteriosa può essere considerato al di sotto di $60 \mathrm{ml} . / \mathrm{min}(1,14)$.

E molto importante nella CIN da somministrazione intra-arteriosa di MDC il concetto di rischio aggiuntivo. In due studi $(8,15)$ gli Autori, dopo aver individuato i singoli fattori di rischio in una popolazione campione, sono stati in grado di formulare uno score di rischio complessivo che è stato validato su una successiva popolazione di pazienti.

La somministrazione endovenosa (e.v.) comporta una minore incidenza di CIN, dovuta sia alla via di somministrazione in sé, che alla minore dose somministrata (oggi nella TAC multistrato può essere inferiore a 100 $\mathrm{mL})$.

Va detto che non sono disponibili delle analisi sui fattori di rischio di CIN dopo somministrazione e.v. di MDC come quelli dei registri cardiologici. Ciò comporta che non sarebbe corretto misurare il rischio dopo una somministrazione e.v. con lo stesso score proposto per le procedure intra-arteriose.

In una recente review sugli studi randomizzati controllati ed osservazionali che hanno considerato l'incidenza complessiva di CIN dopo TAC con MDC, quest'ultima è risultata mediamente del 5.1\% (1.3-21\%) (16).

Kim et al hanno evidenziato che l'incidenza di CIN era rispettivamente del $2.9 \%$ e del $12 \%$ nei pazienti con GFR $30-44 \mathrm{e}<30 \mathrm{~mL} / \mathrm{min}$ sottoposti a TAC con MDC (17).

Nello studio retrospettivo CONNECT su 493 pazienti sottoposti a TAC con MDC (18) l'incidenza di CIN era del 5.9\% nei 169 pazienti con insufficienza renale cronica (GFR misurato con la creatinina clearance $=36.1 \pm 15.7$ $\mathrm{mL} / \mathrm{min}$ ) e saliva al $7.1 \%$ quando l'insufficienza renale era associata a diabete.

Alla luce della minore incidenza dimostrata negli studi sulla somministrazione e.v. le recenti linee guida ESUR (1) concludono che un GFR inferiore a $45 \mathrm{~mL} / \mathrm{min}$ vada considerato come cut off di rischio renale di CIN dopo MDC e.v.

Il fattore di rischio legato alla procedura più importante e più facilmente quantificabile è costituito dalla dose del $\operatorname{MDC}(19,20)$.

Alcuni studi sulla somministrazione intra-arteriosa han-

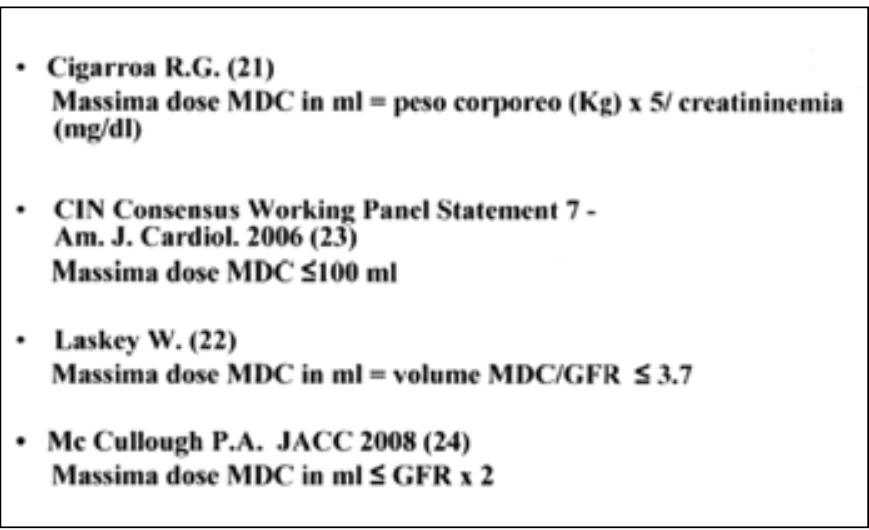

Fig. 1 - Dose massima di MDC rispetto al rischio di sviluppare nefropatia da contrasto nella somministrazione intra-arteriosa. (Vedi riferimenti bibliografici in Bibliografia9.

no individuato come cut off di rischio di CIN la dose di MDC (in $\mathrm{mL}$ ) al di sopra di quella ottenuta dalla formula: peso corporeo (in $\mathrm{kg}$ ) x 5 diviso per il valore di creatinina plasmatica (indice di Cigarroa) $(20,21)$.

La dose considerabile "a rischio" viene ridotta rispetto alla misurazione con il precedente indice se si considera un volume massimo del MDC non eccedente 3.7 volte il filtrato glomerulare misurato con la formula MDRD (22).

Un panel di esperti riuniti in una recente Consensus Conference considera il cut off di $100 \mathrm{~mL}$ per valori di GFR al di sotto di $60 \mathrm{~mL} / \mathrm{min}$ (23).

Un altro indice proponibile potrebbe essere il GFR moltiplicato due volte (24) (Fig. 1).

Va detto che queste considerazioni sulla dose si riferiscono alla somministrazione intra-arteriosa e pertanto non sono applicabili alla somministrazione e.v. rispetto alla quale mancano studi di riferimento.

\section{Conseguenze cliniche della CIN}

Nella maggioranza dei casi la CIN regredisce dopo pochi giorni dalla somministrazione del MDC, tuttavia la sua presenza sembra condizionare negativamente l'outcome successivo del paziente.

Negli studi osservazionali su larga scala in pazienti sottoposti ad angioplastica coronaria (PCI) i pazienti che presentano CIN hanno dimostrato, in analisi univariate, una maggiore mortalità generale e cardiovascolare intra-ricovero e nel successivo follow-up $(7,10,25)$.

In uno studio più recente Goldenberg et al hanno valutato la sopravvivenza a 5 anni di 78 pazienti sottoposti a coronarografia dei quali 10 (13\%) avevano avuto una CIN 
con un recupero della funzione renale dopo 7 giorni. L'analisi Kaplan-Meier mostrava non solo una maggiore mortalità nei pazienti che avevano avuto una CIN, ma anche, e forse più importante, che quelli che non avevano presentato una CIN avevano una perdita di filtrato renale nel follow-up successivo significativamente minore rispetto a quelli che avevano manifestato CIN (26).

Questo dato dell'influenza negativa della CIN sulla funzione renale in un follow-up di 3 anni è stato confermato anche in 520 pazienti sottoposti a TAC (17).

In uno studio retrospettivo di Budano et al su 755 pazienti sottoposti a coronarografie selettive o in urgenza, la presenza di CIN, definita dall'aumento del valore di creatinina $\geq 0.5 \mathrm{mg} / \mathrm{dL}$, rappresenta un fattore di rischio indipendente di decesso a un anno (OR 3.1 C.I. 95\% = 1.2-7.3) (27).

Il problema aperto è comunque capire se la CIN rappresenti l'effetto dello stato di morbidità dei pazienti (soprattutto per problemi cardiovascolari, diabete ed insufficienza renale cronica), oppure rappresenti in sé un fattore di rischio (28).

Questa risposta ci può pervenire solo dal risultato degli studi randomizzati controllati.

Solomon et al hanno rivalutato dopo un anno 294 pazienti che erano stati arruolati nello studio randomizzato CARE osservando che l'incidenza di eventi avversi maggiori (stroke, infarto miocardio, ingresso in dialisi, decesso) era maggiore nei pazienti che avevano presentato una CIN, definita dall'incremento della creatininemia $\geq 0.3 \mathrm{mg} / \mathrm{dL}$ rispetto al valore basale (29).

Più recentemente Briguori et al (6) in uno studio su 410 pazienti sottoposti ad arteriografia coronarica e/o periferica e/o angioplastica hanno dimostrato con una analisi multivariata che la CIN, valutata con un incremento della creatinemia $\geq 0.3 \mathrm{mg} / \mathrm{dL}$ e della cistatina $\mathrm{C}>$ del $10 \%$, rappresentava un rischio indipendente di decesso e di entrata in dialisi nel successivo anno di osservazione, confermando quanto già osservato nella post hoc analisi di Solomon dello studio CARE.

\section{Prevenzione della CIN}

Premesso che la prevenzione nei confronti della CIN deve obbligatoriamente partire dalla individuazione dei soggetti a rischio e che ogni intervento deve essere proporzionato al rischio globale del paziente, diversi provvedimenti sono stati impiegati nella prevenzione della CIN. Lidratazione rappresenta la sola profilassi ad essere strettamente raccomandata dalle Linee Guida (1). L'efficacia della idratazione trova spiegazione nell'azione protettiva dell'espansione del volume circolante nei

\begin{tabular}{|l|l|l|l|}
\hline & Merten (34) & Briguori (35) & Masuda (37) \\
\hline Fisiologica & $5.6 \pm 0.6$ & $5.6 \pm 0.8$ & $5.6 \pm 0.9$ \\
$\begin{array}{l}\text { Sodio } \\
\text { Bicarbonato }\end{array}$ & $6.5 \pm 0.8^{*}$ & $6.6 \pm 0.9^{*}$ & $7.0 \pm 1.2^{*}$ \\
$\approx \mathrm{p}<0.05$ & & & \\
\hline
\end{tabular}

Fig. 2 - pH urinario dopo idratazione con soluzione fisiologica e con Sodio bicarbonato. (Vedi riferimenti bibliografici in Bibliografia).

confronti della vasocostrizione dovuta al MDC e nell'aumento della diuresi che riduce la tossicità diretta da contatto del MDC sulle cellule tubulari renali.

Numerosi studi hanno riguardato la via di somministrazione, la durata ed il tipo di idratazione $(30,31)$.

Per quanto, sia la somministrazione per os, che e.v. (a bolo o continua) si siano dimostrate efficaci (32), i protocolli di prevenzione consigliati dalle Linee Guida ESUR e dagli esperti considerano più efficace nei soggetti a rischio l'idratazione e.v. alla dose di $1-1.5 \mathrm{~mL} / \mathrm{kg} /$ ora a partire da $6-12$ ore prima della somministrazione del MDC e per le successive 6-24 ore $(1,24)$.

Riguardo il tipo di soluzione da infondere uno studio randomizzato ha dimostrato che la soluzione salina isotonica è più efficace rispetto all'ipotonica (33).

Negli ultimi anni la letteratura ha registrato un notevole interesse per l'utilizzo del bicarbonato di sodio (Na bic.). Il Na bic. è stato proposto nella prevenzione della CIN per la sua capacità di ottenere un aumento del $\mathrm{pH}$ urinario osservato in alcuni studi (Fig. 2) che può ridurre la produzione di radicali liberi ossidativi indotti dall'ipossia midollare legata alla vasocostrizione indotta dal MDC.

Il primo studio sul Na bic. si deve a Merten et al (34) che hanno paragonato la fisiologica a una soluzione di $\mathrm{Na}$ bic. 154 molare (con un'infusione rapida iniziale di $3 \mathrm{~mL} / \mathrm{kg} / \mathrm{min}$ per un'ora seguita da $1 \mathrm{~mL} / \mathrm{kg}$ /ora per 6 ore) in 119 pazienti con insufficienza renale moderata (GFR $41-45 \mathrm{~mL} / \mathrm{min}$ ) sottoposti a somministrazione di MDC intra-arterioso o e.v. L'Na bic. ha ottenuto una minore incidenza di CIN (1.7\% vs. $13.6 \%$; $\mathrm{p}<0.001)$.

Successivamente sono stati pubblicati numerosi altri studi prospettici randomizzati, per la maggioranza su pazienti a rischio sottoposti a procedure intra-arteriose, affetti da insufficienza renale moderata (GFR $60-40 \mathrm{~mL} /$ min) con protocolli differenti (per dose, tempi di infusione ed associazione con altre terapie preventive) rispetto a quello utilizzato nello studio originale. 


\begin{tabular}{|c|c|c|c|c|}
\hline Autore & $\mathrm{N}$ trials & $\begin{array}{l}\mathrm{N} \\
\text { pazienti }\end{array}$ & $\begin{array}{l}\text { RR } \\
\text { (I.C. } 95 \% \text { ) }\end{array}$ & $\begin{array}{l}\text { Eterogeneità } \\
\text { Statistica } \\
\text { (p) }\end{array}$ \\
\hline Hogan S.(59) & 7 & 1307 & $\begin{array}{l}0.37 \\
(0.18-0.71)\end{array}$ & $\begin{array}{l}\text { Presente } \\
(0.009)\end{array}$ \\
\hline Brar S.(60) & $\begin{array}{l}\text { Tot. } 14 \\
\text { "High } \\
\text { quality } 8\end{array}$ & $\begin{array}{l}2290 \\
1890\end{array}$ & $\begin{array}{l}\text { Non } \\
\text { riportata } \\
0.71 \\
(0.43-1.03)\end{array}$ & 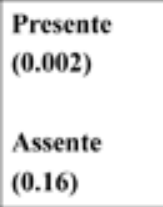 \\
\hline Meier P.(61) & 17 & 2633 & $\begin{array}{l}0.52 \\
(0.34-0.80)\end{array}$ & $\begin{array}{l}\text { Presente } \\
(0.015)\end{array}$ \\
\hline Hoste E.(40) & 18 & 3055 & $\begin{array}{l}0.66 \\
(0.45-0.95)\end{array}$ & $\begin{array}{l}\text { Presente } \\
(0.005)\end{array}$ \\
\hline Trivedi H.(62) & 10 & 1090 & $\begin{array}{l}0.57 \\
(0.38-0.85) \\
\end{array}$ & $\begin{array}{l}\text { Assente } \\
(0.10)\end{array}$ \\
\hline Kunadian V.(63) & 7 & 1734 & $\begin{array}{l}0.33 \\
(0.16-0.69)\end{array}$ & $\begin{array}{l}\text { Presente } \\
(0.001)\end{array}$ \\
\hline
\end{tabular}

Fig. 3 - Meta analisi sull'utilizzo della idratazione con bicarbonato di sodio rispetto alla fisiologica nella prevenzione della CIN. (Vedi riferimenti bibliografici in Bibliografia).

Tra gli studi più citati ve ne sono alcuni con risultati positivi (35-37) ed altri con risultati negativi $(38,39)$.

Per chiarire meglio il ruolo del $\mathrm{Na}$ bic. gli studi sono stati aggregati in diverse meta-analisi comprendenti lavori a stampa ed abstract a convegni.

In quasi tutte le meta-analisi il risultato aggregato mostra una riduzione del rischio di CIN nei protocolli con $\mathrm{Na}$ bic., che però si associa alla presenza di una significativa eterogeneità statistica (che indica quanto il risultato di ogni singolo trial si discosti dal risultato aggregato) (Fig. 3) .

Una delle più recenti meta-analisi (40) ha analizzato 3055 pazienti in 18 studi: nella maggioranza degli studi i pazienti avevano un grado di insufficienza renale valutato con $\mathrm{GFR}<60 \mathrm{~mL} / \mathrm{min}$; le procedure erano solo in 13 studi intra-arteriose e negli altri 5 anche e.v. In 10 trial veniva utilizzata anche la N-Acetilcisteina (NAC), mentre il protocollo di infusione e.v. era identico a quello di Merten (34) in 9 trial ed era differente in 4 studi.

Il dato aggregato ha mostrato una significativa riduzione del rischio di CIN con Na bic. (RR 0.66; IC $95 \%=0.45$ $0.95)$. Va però sottolineato che l'eterogeneità della metaanalisi era statisticamente significativa $(52 \% ; \mathrm{p}=0.005)$. Il dato interessante è che gli studi che dimostrano una maggiore efficacia del $\mathrm{Na}$ bic. sono accomunati da un protocollo di infusione rapida prima della procedura.

Questo dato è stato recentemente confermato da una meta-analisi di Meier (41) e porterebbe a dire che l'Na bic. risulta più utile della fisiologica quando non c'è tempo di eseguire un'idratazione prolungata e quindi potrebbe essere più indicato nelle procedure in emergenza (angioplastica primaria).

La maggiore efficacia dell'idratazione con $\mathrm{Na}$ bic. rispetto alla fisiologica nelle procedure di angioplastica primaria in pazienti con infarto miocardico STEMI è stata recentemente osservata da Maioli et al in uno studio randomizzato su 450 pazienti (GFR medio $76 \pm 20 \mathrm{~mL} /$ min). Il gruppo trattato con Na bic. alla dose di $3 \mathrm{~mL} /$ $\mathrm{kg}$ per un'ora seguito da $1 \mathrm{~mL} / \mathrm{kg}$ per 12 ore dopo la procedura ha presentato un'incidenza di CIN significativamente minore (12\%) rispetto al gruppo che eseguiva idratazione con fisiologica solo per le 12 ore seguenti la procedura (22.7\%) e al gruppo di controllo senza idratazione (27\%). Va considerato però che il volume di idratazione era maggiore nel gruppo che eseguiva $\mathrm{Na}$ bic. rispetto a quello trattato con fisiologica (42).

Abbiamo detto precedentemente come il ruolo protettivo dell'idratazione sia riconducibile sia all'espansione del volume, che all'aumento della diuresi.

L'effetto della diuresi forzata (ottenuta dall'idratazione e.v. associata a dopamina e diuretico) è stato dimostrato dallo studio PRINCE su 98 pazienti con elevato rischio renale (GFR $31.8 \pm 11.6 \mathrm{~mL} / \mathrm{min}$.) sottoposti a PCI. Lincidenza di CIN era significativamente maggiore (41\%) nei pazienti con una diuresi $\leq 150 \mathrm{~mL} /$ ora, rispetto a quelli con una diuresi $>150 \mathrm{~mL} /$ ora $(16.2 \%) \quad(\mathrm{p}<0.05)$ (43) .

Più recentemente nello studio MYTHOS su 170 pazienti con GFR $39 \pm 10 \mathrm{~mL} / \mathrm{min}$ sottoposti a procedure coronariche, Marenzi et al (44) hanno utilizzato il sistema Renal Guard (RG) nel quale viene ottenuta una diuresi forzata con diuretico e.v. (> $300 \mathrm{~mL} /$ ora da un'ora prima della somministrazione di MDC e per 4 ore successive) mantenendo un bilancio IN/OUT in pareggio attraverso un'infusione e.v. automatizzata bilanciata alla diuresi del paziente. L'incidenza di CIN è stata del 18\% nel gruppo di controllo trattato con sola idratazione e.v. rispetto al $4.6 \%$ in quelli con $\mathrm{RG}(\mathrm{p}=0.005)$.

La N-Acetilcisteina (NAC) è stata utilizzata in molti trial sulla prevenzione della CIN in soggetti a rischio (45). Tepel et al hanno per primi dimostrato l'efficacia della NAC nella riduzione della CIN ( 2 vs $21 \% ; \mathrm{p}=0.01$ ) (con una dose per os di $600 \mathrm{mg}$ due volte al di) in un gruppo di 83 pazienti con insufficienza renale moderata/severa (creatininemia $2.4 \pm 1.3 \mathrm{mg} / \mathrm{dL}$ ) sottoposti a TAC con MDC (46).

Gli studi successivi non sono stati sempre in grado di confermare questo risultato per cui, come nel caso del $\mathrm{Na}$ bic., sono stati aggregati in diverse meta analisi. La maggior parte di esse ha potuto dimostrare una signifi- 


\begin{tabular}{|l|l|l|l|l|}
\hline Autore & N trials & N pazienti & $\begin{array}{l}\text { RR } \\
(1 . C .95 \\
\%)\end{array}$ & $\begin{array}{l}\text { Eterogeneità } \\
\text { statistica } \\
(p)\end{array}$ \\
\hline Kshirsagar A. (64) & 16 & 1538 & $\begin{array}{l}\text { Non } \\
\text { riportata }\end{array}$ & $\begin{array}{l}\text { Presente } \\
(<0.001)\end{array}$ \\
\hline Pannu N. (65) & 15 & 1776 & $\begin{array}{l}0.65 \\
(0.43-1)\end{array}$ & $\begin{array}{l}\text { Presente } \\
(0.02)\end{array}$ \\
\hline Bagshaw S. (66) & 14 & 1261 & $\begin{array}{l}0.54 \\
(0.32-0.91)\end{array}$ & $\begin{array}{l}\text { Presente } \\
(0.03)\end{array}$ \\
\hline Nallamothu B. (69) & 20 & 2195 & $\begin{array}{l}0.73 \\
(0.52-1)\end{array}$ & $\begin{array}{l}\text { Presente } \\
(0.08)\end{array}$ \\
\hline Liu R. (67) & 9 & 1028 & $\begin{array}{l}0.43 \\
(0.24-0.75)\end{array}$ & $\begin{array}{l}\text { Presente } \\
(0.03)\end{array}$ \\
\hline Duong M. (68) & 14 & 1584 & $\begin{array}{l}0.57 \\
(0.37-0.84)\end{array}$ & $\begin{array}{l}\text { Presente } \\
(0.01)\end{array}$ \\
\hline
\end{tabular}

Fig. 4 - Meta analisi sull'utilizzo della $\mathrm{N}$-acetilcisteina nella prevenzione della nefropatia da contrasto. (Vedi riferimenti bibliografici in Bibliografia).

cativa riduzione del rischio di CIN con NAC (47), sebbene fosse presente una significativa eterogeneità sia metodologica, che statistica (Fig. 4).

Il fattore metodologico più critico è rappresentato dalla differenza nella dose di NAC somministrata nei vari studi.

L'utilizzo di una dose orale giornaliera di $2400 \mathrm{mg}$ di NAC per due giorni si è dimostrata più efficace rispetto a $1200 \mathrm{mg}$ (associate ad idratazione e.v.) nella angiografia coronarica o periferica in pazienti con creatinina clearance $<60 \mathrm{~mL} / \mathrm{min}$ (incidenza di CIN 3.5\% vs $11 \%$ ) (p $=0.038)(48)$.

Marenzi et al hanno dimostrato la maggiore efficacia (incidenza di CIN $8 \%$ vs $15 \%$; $\mathrm{p}=0.02$ ) di una dose elevata (1200 e.v seguita da $2400 \mathrm{mg} /$ die per os per due giorni), rispetto a $600 \mathrm{mg}$ e.v seguiti da $1200 \mathrm{mg} /$ die per os in uno studio clinico randomizzato su 354 pazienti con IMA sottoposti ad angioplastica primaria con modesta insufficienza renale (creatinina clearance $77 \pm 25 \mathrm{~mL}$ / $\min (49)$.

Questo risultato nei casi di angioplastica primaria non si è confermato in uno studio più recente su 251 pazienti con una funzione renale migliore $(85 \mathrm{~mL} / \mathrm{min})(50)$.

Limportanza della dose è stata confermata da una recente meta-analisi (51) di 16 studi randomizzati su 1677 pazienti (creatininemia $1.58 \mathrm{mg} / \mathrm{dL}, \mathrm{GFR}<60 \mathrm{~mL} / \mathrm{min}$; $38.7 \%$ diabetici) che ha evidenziato una riduzione significativa del rischio (OR 0.46 I.C. 95\% = 0.33-0.63) con dosi di NAC di 2400-6000 $\mathrm{mg} /$ die rispetto a dosi inferiori. In questa meta-analisi la qualità metodologica, al contrario delle precedenti, non mostrava bias significativi, così come l'eterogeneità statistica non era significativa.
Dopo questa meta-analisi è stato pubblicato lo studio ACT dal quale si attendeva una parola definitiva sull'efficacia della NAC (52). L'ACT è uno studio randomizzato su una ampia popolazione di pazienti brasiliani (2308) sottoposti ad angiografia coronarica o periferica che confronta la NAC, eseguita alla dose di $2400 \mathrm{mg}$ per os il giorno prima e il giorno dopo l'esame associata ad idratazione e.v., alla sola idratazione e.v. L' incidenza di CIN è stata del $12.7 \%$ nei due gruppi.

Il risultato negativo sull'efficacia della NAC va però letto alla luce di alcuni limiti metodologici, quali l'utilizzo di un protocollo di idratazione non rigoroso della durata complessiva di 12 ore, e l'utilizzo di differenti MDC, nel $22 \%$ di tipo iperosmolare (che è stato tolto da tempo dal commercio in Europa e in USA).

Un punto critico riguarda, inoltre, la popolazione studiata che presentava un ridotto rischio renale (GFR medio di $69 \pm 27 \mathrm{~mL} / \mathrm{min}$ ) (solo il $4.6 \%$ di pazienti con un $\mathrm{GFR}<30 \mathrm{~mL} / \mathrm{min})$ e la dose di MDC, $100 \mathrm{~mL}(70$ $130 \mathrm{~mL}$ ), che era inferiore rispetto alla maggior parte degli studi nei quali la NAC si è dimostrata efficace.

Tutto quanto è stato detto in tema di prevenzione (idratazione, NAC, Na bic.) si è dimostrato efficace nei pazienti con un rischio renale moderato (GFR 40-59 mL/ $\mathrm{min}$ ); il problema riguarda quale terapia adottare nei pazienti con grave insufficienza renale preesistente (GFR $<30 \mathrm{~mL} / \mathrm{min}$ ) nei quali l'utilizzo del MDC rappresenta un rischio di peggioramento ulteriore e di ingresso in dialisi.

In questi casi è stata valutata l'efficacia dell'emodialisi e dell'emofiltrazione.

L'emodialisi, in particolare quando viene condotta con membrane ad elevata permeabilità, è in grado di rimuovere dal circolo il MDC e di ridurne le concentrazioni plasmatiche, tuttavia il risultato sulla prevenzione della CIN è stato molto spesso negativo (53).

Tra gli studi positivi sull'utilizzo dell'emodialisi vi è quello di Lee et al (54) che hanno valutato 82 pazienti sottoposti a coronarografia con creatinina clearance $13 \pm 4$ $\mathrm{mL} / \mathrm{min}$; i pazienti sono stati randomizzati ad emodialisi con filtro in polisulfone di 4 ore, eseguita dopo la procedura, associata ad idratazione 6 ore prima e 12 ore dopo, o a sola idratazione e.v. con le stesse modalità. Il gruppo che ha eseguito emodialisi ha evidenziato una minore incidenza di CIN (5\% vs 35\%), di mortalità intra-ricovero (0 vs $13 \%)$ e necessità di dialisi cronica (2\% vs $35 \%$ ) (un paziente vs 14) nel successivo follow-up.

Va sottolineato che in questo trial la dose di MDC nel gruppo trattato con dialisi $(106.8 \pm 44 \mathrm{~mL})$ era mediamente inferiore rispetto a quella di altri studi che hanno fornito risultati negativi (53).

Rispetto alla dialisi l'emofiltrazione veno-venosa conti- 
nua (CVVH) ha dato risultati migliori nella prevenzione della CIN. Questi risultati possono dipendere dalla peculiarità di questo trattamento che ottiene una minore riduzione della volemia e della gittata cardiaca rispetto all'emodialisi convenzionale (53) e nella maggiore stabilità emodinamica durante il trattamento.

Marenzi et al hanno dimostrato la maggiore efficacia dell'emofiltrazione $\mathrm{CVVH}$ rispetto alla idratazione e.v in 114 pazienti sottoposti a PCI con creatinina clearance di $26 \pm 8 \mathrm{~mL} / \mathrm{min}$ (25\% con frazione di eiezione ventricolare sinistra $<40 \%$ ) (incidenza di CIN $5 \%$ vs $50 \%$; $\mathrm{p}<$ 0.001 ; necessità di dialisi nel $3 \%$ (2 pazienti) vs $25 \%$ (14 pazienti) (55).

In uno studio successivo lo stesso gruppo ha paragonato tre differenti protocolli in 92 pazienti con creatinina clearance di $19 \pm 4 \mathrm{~mL} / \mathrm{min}$. Tutti i pazienti hanno eseguito una idratazione pre procedura e.v. ( $1 \mathrm{~mL} / \mathrm{kg}$ /ora). Il gruppo di controllo proseguiva l'idratazione per le successive 12 ore, nel secondo gruppo veniva eseguita CVVH per 18-24 ore solo dopo e nel terzo 6 ore prima e 18-24 ore dopo. L'incidenza di CIN era significativamente inferiore $(\mathrm{p}=0.0013)$ nel terzo gruppo $(3 \%)$ rispetto al controllo (40\%) e al gruppo trattato con CVVH dopo la procedura (26\%). La dialisi per insufficienza renale grave si era eseguita in 9 pazienti (30\%) nel gruppo di controllo, in $3(10 \%)$ nel gruppo trattato con CVVH post- ed in nessun paziente nel gruppo che aveva eseguito $\mathrm{CVVH}$ pre- e post- (56).

L'emofiltrazione è stata utilizzata in associazione alla NAC e all'idratazione e.v in uno studio prospettico su 23 pazienti ad elevato rischio (GFR $17.3 \pm 5 \mathrm{~mL} / \mathrm{min}, 11$ diabetici, 11 con segni di scompenso cardiaco). I pazienti sono stati trattati dopo angiografia o PCI con CVVH per 6 ore durante le quali veniva eseguita una extra idratazione in vena periferica di 2 litri di fisiologica, con un bilancio liquido in/out in pareggio durante il trattamento. Una idratazione e.v $(1.2-1.6 \mathrm{~mL} / \mathrm{min})$ veniva eseguita per 12 ore prima e 24 ore dopo. Lincidenza di CIN è stata del $9 \%$. In tutti i pazienti non si è osservato peggioramento della funzione renale alla dimissione rispetto al basale (GFR $20 \pm 7 \mathrm{~mL} / \mathrm{min}$ ) ed in nessun caso è stato necessario ricorrere alla dialisi (57).

Recentemente nei pazienti ad alto rischio renale è stato utilizzato il sistema Renal Guard (RG).

Briguori et al nello studio REMEDIAL II (58) hanno valutato 292 pazienti con insufficienza renale (GFR 32 $\pm 8 \mathrm{~mL} / \mathrm{min}$ ) associata ad altri fattori di rischio di CIN (Mehran score $12 \pm 2$ ) sottoposti a angiografia coronaria o periferica e/o angioplastica. I pazienti sono stati randomizzati in due gruppi. Il gruppo di controllo veniva trattato con idratazione con Na bic. $154 \mathrm{mMol} / \mathrm{L}$ per un'ora prima della somministrazione del MDC alla dose di $3 \mathrm{~mL} / \mathrm{kg} /$ ora e per 6 ore dopo alla dose di 1 $\mathrm{mL} / \mathrm{kg} /$ ora. Il secondo gruppo eseguiva il sistema $\mathrm{RG}$ con una diuresi media di $352 \pm 131 \mathrm{~mL}$, dopo bolo e.v di fisiologica $250 \mathrm{~mL}$ e diuretico (dose media $14 \pm 8 \mathrm{mg}$ e.v. ) bilanciata automaticamente da una reinfusione con fisiologica, per 5 ore e 75 min (range 3-9 ore). Entrambi i gruppi assumevano NAC prima e dopo il MDC. L'incidenza di CIN, valutata come aumento della creatinina $\geq 0.3 \mathrm{mg} / \mathrm{dL}$, era significativamente ridotta nel gruppo che eseguiva il RG rispetto al gruppo trattato solo con idratazione (rispettivamente $11 \%$ vs $20.5 \%$ (OR 0.47; IC 95\% 0.24-0.92). Inoltre in 6 pazienti nel gruppo di controllo rispetto a solo uno nel gruppo trattato con RG si è verificata insufficienza renale acuta con necessità di dialisi (OR 0.16; I.C. 95\% 0.02-1.13).

\section{Conclusioni}

In conclusione la prevenzione della CIN deve prevedere: - lo screening dei pazienti a rischio per presenza di insufficienza renale valutando la funzione renale con la formula MDRD e considerando un cut off di GFR di $60 \mathrm{~mL} / \mathrm{min}$ per la somministrazione intra-arteriosa e di $45 \mathrm{~mL} / \mathrm{min}$ per quella venosa.

Un questionario che individui la possibile presenza di nefropatia può essere molto utile nelle PCI primarie in assenza di dati di funzione renale.

- La dose di MDC nella somministrazione intra-arteriosa deve essere la minore possibile con un cut off nel paziente a grave rischio di CIN (GFR $\leq 30 \mathrm{~mL} / \mathrm{min}$ ) di $100 \mathrm{~mL}$. In alternativa si potrebbe utilizzare la formula:

$$
\text { dose massima di MDC (ml) = GFR x } 2
$$

È consigliabile, nei pazienti a rischio, intervallare la diagnostica e la interventistica cardiaca di 72 ore.

- Lidratazione va eseguita da 12 ore prima del MDC e per almeno 24 ore alla dose di 1.0. - $2 \mathrm{~mL} / \mathrm{kg} /$ ora in base alle condizioni cliniche. Va considerata l'importanza nei pazienti ad alto rischio di ottenere una diuresi elevata ( $\geq 150 \mathrm{~mL} /$ ora) e pertanto di adeguare la dose diuretica e l'idratazione a questo scopo.

- Il sodio bicarbonato $154 \mathrm{Mol}$ è consigliabile, secondo il protocollo di Merten (34), nelle PCI primarie in essenza del valore di funzione renale.

- Nei pazienti a rischio la NAC è consigliabile a dosi $1200 \mathrm{mg}$ due-tre volte al dì per os dalle 24 ore precedenti e per le successive $24-48$ ore.

- L'emofiltrazione CVVH deve essere riservata ai pazienti con GFR $<30 \mathrm{~mL} / \mathrm{min}$ sottoposti a somministrazione intra-arteriosa di MDC o in casi selezionati ad alto rischio (scompenso cardiaco, dosi elevate di MDC). 

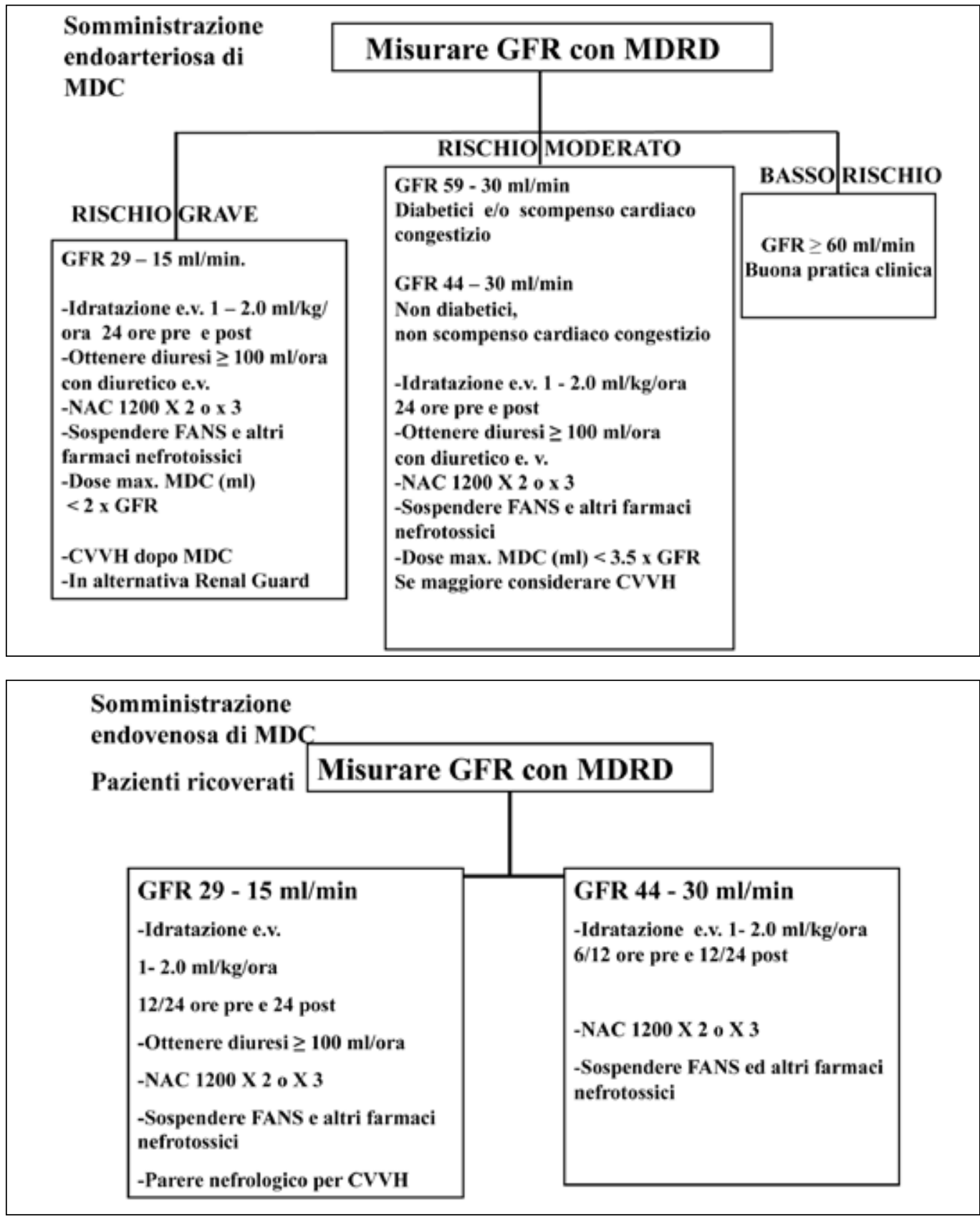

Fig. 5 - Algoritmo per la prevenzione della nefropatia da contrasto dopo somministrazione intra- arteriosa utilizzato nell'A.O. Ospedale Civile di Legnano. CVVH = emofiltrazione veno-venosa continua. NAC $=\mathbf{N}$-Acetilcisteina.
Fig. 6 - Algoritmo per la prevenzione della nefropatia da contrasto dopo somministrazione endovenosa in pazienti ricoverati utilizzato nell'A.O. Ospedale Civile di Legnano. CVVH = emofiltrazione veno - venosa continua. $\mathbf{N A C}=\mathbf{N}$-Acetilcisteina.
- Tutti i farmaci potenzialmente nefrotossici devono essere possibilmente evitati.

La furosemide può essere mantenuta (o aumentata) in corso di idratazione nei pazienti con IRC o scompenso cardiaco. Il diuretico è da sospendere solo se viene utilizzato a scopo antiipertensivo, ma non nei pazienti con scompenso che devono eseguire idratazione e.v.

Infine va valutato l'effetto del diuretico in relazione alla possibilità che si debba ottenere una diuresi oraria efficace (150-200 mL /ora) nei pazienti a maggiore rischio di CIN.

- Nei pazienti con elevato rischio di CIN in alternativa alla CVVH può essere proponibile l'utilizzo del sistema
Renal Guard secondo i protocolli utilizzati negli studi citati di Marenzi et al e di Briguori et al $(44,58)$.

- Sulla scorta di queste considerazioni il protocollo di prevenzione della CIN deve essere stratificato sia in base al rischio del paziente, determinato dal grado di insufficienza renale e dalle condizioni cliniche di rischio aggiuntivo (diabete, scompenso cardiaco, dosi elevate di MDC), che alla modalità di somministrazione (intra arteriosa o endovenosa).

Le Figure 5 e 6 mostrano lo schema di protocollo di prevenzione della CIN costruito su questi criteri in uso nel nostro ospedale. 


\section{Riassunto}

La nefropatia da mezzo di contrasto (contrast induced nephropathy) (CIN) è una delle cause più frequenti di danno renale acuto nei pazienti ricoverati.

L'incidenza di CIN dipende dalla presenza di fattori di rischio legati al paziente (insufficienza renale, diabete, malattie cardiovascolari, età avanzata) e da cause dipendenti dalla procedura (dose elevata di mezzo di contrasto, via di somministrazione intra-arteriosa). L'insufficienza renale rappresenta il maggiore fattore di rischio di CIN, in particolare quando è associata al diabete.

Lidratazione pre- e post-somministrazione di MDC rappresenta la sola terapia di prevenzione ad essere strettamente raccomandata dalle Linee Guida nei pazienti a rischio.

Gli studi sulla prevenzione della CIN hanno riguardato soprattutto casistiche cardiologiche di pazienti con moderato rischio renale (GFR $60-40 \mathrm{~mL} / \mathrm{min}$ ) sottoposti a somministrazione intra-arteriosa di mezzo di contrasto. In molti trial clinici è stata valutata l'efficacia dell'idratazione con bicarbonato di sodio e della $\mathrm{N}$-acetilcisteina (NAC) nella prevenzione della CIN.

Linfusione con sodio bicarbonato ha dimostrato una maggiore efficacia rispetto alla fisiologica, in modo particolare quando l’idratazione necessita tempi rapidi come nelle procedure in emergenza.

La NAC non ha dimostrato una chiara efficacia in quanto i risultati positivi osservati in alcuni studi non sono stati confermati in altri.

Il problema aperto rimane la prevenzione della CIN nei soggetti con elevato rischio renale (eGFR $<30 \mathrm{~mL} / \mathrm{min}$ ) nei quali la presenza di CIN può associarsi all'ingresso in dialisi cronica.

Parole Chiave. Contrasto, $\mathrm{N}$-acetilcisteina, Insufficienza renale acuta

\section{Bibliografia}

1. Stacul F, Van der Molen A, Reimer P, et al. Contrast induced nephropathy: updeted ESUR Contrast Media Safety Commitee guidelines. Eur Radiol 2011; 21: 2527-41.

2. Mc Cullough P. Contrast induced acute kidney injury. JACC 2008; 51: 1419-28.

3. Murray P, Palevsky P. Acute kidney injury and critical care nephrology. NephSAP 2009; 8: 175-8.

4. Coca SG, Yalavarthy R, Concato J, et al. Biomarkers for the diagnosis and risk stratification of acute kidney injury: a systematic review. Kidney Int 2008; 73: 1008-16.

5. Malyszko J. Biomarkers of acute kidney injury in different clinical setting: a time to ch'ange the paradigm? Kidney Blood Press Res 2010; 33: 368-82.

6. Briguori C, Visconti G, Rivera N, et al. Cystatin C and contrast induced acute kidney injury. Circulation 2010; 121: 2117-22.

7. Rihal CS, Textor S, Grill DE, et al. Incidence and prognostic importance of acute renal failure after percutaneous coronary intervention. Circulation 2002; 105: 2259-64.

8. Meheran R, Aymong ED, Nikolsky E, et al. A simple risk score for prediction of contrast induced nephropathy after percutaneous coronary intervention. JACC 2004; 44: 1393-9.

9. Mc Cullough P, Adam A, Becker C, et al. Risk prediction of contrast induced nephropathy. Am J Cardiol 2006; 98 (Suppl 6): S27-36.

10. Dangas G, Iakovou I, Nikolsky E, et al. Contrast induced nephropathy after percutaneous coronary interventions in relation to chronic kidney disease and hemodynamic variables. Am J Cardiol 2005; 95: 13-9.

11. National Kidney Foundation K/DOQI clinical practice guidelines for chronic kidney disease: evaluation, classification, and stratification. Am J Kidney Dis 2002; 39 (Suppl 2): S1-66.

12. Thomsen H. European Society of Urogenital Radiology Guidelines on contrast media application. Curr Opinion Urol 2007; 17: 70-6.

13. Levey A, Stevens L, Schmid C, et al. A new equation to estimate glomerular filtration rate. Ann Intern Med 2009; 150: 604-12.

14. Lameire R, Adam A, Becker C, et al. Baseline renal function screening. Am J Cardiol 2006; 98(Suppl 6): S20-6.

15. Skelding KA, Bartholomew B, O'Neill W. Validation of a predictive risk score for radiocontrast-induced nephropathy following percutaneous coronary intervention. J Invasive Cardiol 2007; 19: 229-33.

16. Katzberg R, Lamba R. Contrast induced nephropathy after intravenous administration: fact or fiction? Rad Clin $\mathrm{N}$ Am 2009; 47: 789-800.

17. Kim SM, Cha R, Lee JP, et al. Incidence and outcomes 
of contrast induced nephropathy after computed tomography in patients with CKD: a quality improvement report. Am J Kidney Dis 2010; 55: 1018-25.

18. Leoncini R, Fattori R, Morana G, et al. Contrast induced nephropathy in patients undergoing computer tomography (CONNECT) - A clinical problem in daily practice? A multicenter observational study. Acta Radiol 2010; 7: 741-50.

19. Marenzi G, Assanelli E, Campodonico J, et al. Contrast volume during primary percutaneous coronary intervention and subsequent contrast induced naphrpathy and mortality. Ann Intern Med 2009; 150: 170-7.

20. Brown JR, Robb JF, Block CA, et al. Does safe dosing of iodinated contrast prevent contrast induced acute kidney injury? Circ Cardiovasc Interv 2010; 3: 346-50.

21. Cigarroa RG, Lange RA, Williams RH, et al. Dosing of contrast material to prevent contrast nephropathy in patients with renal disease. Am J Med 1989; 86: 649- 52.

22. Laskey WK, Jenkins C, Selzer F, et al. Volume to creatinine clearance ratio: a pharmacokinetically based risk factor for prediction of early creatinine increase after percutaneous coronary intervention. JACC 2007; 50: 584-90.

23. Davidson C, Stacul F, MC Cullough P, et al. Contrast medium use. Am J Cardiol 2006; 98(Suppl 6): S43-58.

24. Mc Cullough P. Contrast induced acute kidney injury JACC 2008; 51: 1420-8.

25. 25) Weisbord S, Chen H, Stone R, et al. Associations of increase in serum creatinine with mortality and length of hospital stay after coronary angiography. JASN 2006; 17 : 2871-7.

26. Goldenberg I, Chonchol M, Guetta V. Reversibile acute kidney injury following contrast exposure and the risk of long term mortality. Am J Nephrol 2009; 29: 136-44.

27. Budano C, Levis M, D'Amico M, et al. Impact of contrast induced acute kidney injury definition on clinical outcomes. Am. Heart 2011; 161: 963-71.

28. Mehran R, Brar S, Dangas G. Contrast induced acute kidney injury JACC 2010; 55: 2210-1.

29. Solomon R, Mehran R, Natarajan M, et al. Contrast induced nephropathy and long term adverse events: cause and effect? Clin. J Am Soc Nephrol 2009; 4: 1162-9.

30. Laville M, Juillard L. Contrast induced acute kidney injury: how should at risk patients be identified and managed? J Nephrol 2010; 23: 387-98.

31. Solomon R. Contrast induced acute kidney injury. Circulation 2010; 122: 2451-5.

32. Trivedi H, Moore H, Nasr S, et al. A randomized prospective trial to assess the role of saline hydration on the development of contrast nephropathy. Nephron Clin Pract 2003; 93: C29-C34.

33. Mueller C, Buerkle G, Buettner HJ, et al. Prevention of contrast media associated nephropathy: randomized comparison of 2 hydration regimen in 1620 patients undergoing coronary angioplasty. Arch Intern Med 2002; 162: 329-36.

34. Merten G, Burgess W, Gray L, et al. Prevention of contrast induced nephropathy with sodium bicarbonate. A randomized controlled trial. JAMA 2004; 291: 2328-34.

35. Briguori C, Airoldi F, D'Andrea D, et al. Renal Insufficiency following contrast media administration trial (REMEDIAL): a randomized comparison of 3 preventive strategies. Circulation 2007; 115: 1211-7.

36. Recio-Mayoral A, Chaparro M, Prado B, et al. The reno protective effect of hydration with sodium bicarbonato plus $\mathrm{N}$-acetylcysteine in patients undergoing emergency percutaneous coronary intervention. The RENO study JACC 2007; 49: 1283-8.

37. Masuda M, Yamada T, Mine T, et al. Comparison of usefulness of sodium bicarbonato versus sodium chloride to prevent contrast induced nephropathy in patients undergoing an emergent coronary procedure. Am J Cardiol 2007; 100: 781-6.

38. Maioli M, Toso A, Leoncini M, et al. Sodium bicarbonato versus saline for the prevention of contrast induced nephropathy in patients with renal dysfunction undergoing coronary angiography or interventio. JACC 2008; 52: 599-604.

39. Brar S, Yuh-Jer Shen A, Jorgensen M, et al. Sodium bicarbonate vs sodium chloride for the prevention of contrast medium induced nephropathy in patients undergoing coronary angiography. JAMA 2008; 300: 1038-46.

40. Hoste E, De Waele J, Gevaert S, et al. Sodium bicarbonate for prevention of contrast - induced acute kidney injury:a systematic review and meta-analysis: Nephrol. Dial Transplant 2010; 25: 747-58.

41. Meier P, Gurm H. Is simpler also better? Brief sodium bicarbonate infusion to prevent contrast induced nephropathy. Am J Cardiol 2009; 1042-3.

42. Maioli M, Toso A, Leoncini M, et al. Effects of Hydration in contrast induced acute kidney injury after primary gioplasty. Circulation 2011; 4: 456-62.

43. Stevens M, McCullough P, Tobin K, et al. A prospective randomized trial of prevention measures in patients at high risk for contrast nephropathy. Results of PRINCE study. JACC 1999; 33: 403-11.

44. Marenzi G, Ferrari C, Marana C, et al. Prevention of contrast nephropathy by furosemide with matched hydration. The Mythos trial JACC: cardiovascular interventions 2012; 5: 90-7.

45. Fishbane S. N-acetylcisteine in the prevention of contrast induced nephropathy. Clin J Am Soc Nephrol 2008; 3: 281-7.

46. Tepel M, Van der Giet M, Schwarzfeld C, et al Prevention of radiographic contrast agent induced reductions renal 
function by acetylcysteine. N Engl J Med 2000; 343: 180-4.

47. Marenzi G. La prevenzione della nefropatia da contrasto. G Ital Cardiol 2009; 10: 88-96.

48. Briguori C, Colombo A, Violante A, et al. Standard vs double dose of $\mathrm{N}$-acetylcysteine to prevent contrast agent associated nephroptxicity. Eur Heart J 2004; 25: 206-21.

49. Marenzi G, Assanelli E, Marana I, et al. N-acetylcysteine and contrast induced nephropathy in primary angioplasty. NEJM 2006; 354: 2773-82.

50. Thiele H, Hildebrand L, Schirdewahn C, et al. Impact of high dose N-Acetylcysteine versus placebo on contrast induced nephropathy and myocardial reperfusion injury in unselected patient with ST-segment elevation myocardial infarction undergoing primary percutaneous coronary intervention. J Am Coll Cardiol 2010; 55: 2201-09.

51. Trivedi H, Daram S, Szabo A, et al High dose N-Acetylcysteine for the prevention of contrast induced nephropathy Am J Med 2009;122: 874-80.

52. Berwanger O, Cavalcanti A, Sousa A, et al. Acetylcysteine for prevention of renal outcomes in patients undergoing coronary and peripheral vascular angiography. Results from the randomized acetylcysteine for contrast induced nephropathy trial (ACT). Circulation 2011; 124: 1250-9.

53. Guastoni C, De Servi S, D'Amico M. The role of dialisi in contrast induced nephropathy: doubts and certainties. J Cardiov Med 2007; 8: 549-57.

54. Lee PT, Chou KJ, Liu CP, et al. Renal protection for coronary angiography in advanced renal failure patients by prophylactic hemodialysis. A randomized controlled trial. J Am Coll Cardiol 2007; 50: 1015-20.

55. Marenzi G, Marana I, Lauri G, et al. The prevention of contrast agent induced nephropathy by hemofiltration. $\mathrm{N}$ Engl J Med 2003; 349: 1333-40.

56. Marenzi G, Laurio G, Campodonico J, et al. Comparison of two hemofiltration protocols for prevention of contrast induced nephropathy in high risk patients. Am J Med 2006; 119: 155-62.

57. Guastoni C, Bellotti N, Poletti F, et al. Efficacia della emofiltrazione veno-venosa continua (CVVH) associata a contemporanea idratazione endovenosa nella prevenzione della nefropatia da contrasto (NC) in pazienti ad alto rischio. G Ital Nefrol 2009; S-47: S86.

58. Briguori C, Visconti G, Focaccio A, et al. Renal insufficiency after contrast media administration Trial II (RE-
MEDIAL II) Renal Guard system in high risk patients for contrast induced acute kidney injury. Circulation 2011; 124: $1260-9$.

59. Hogan S, LAAllier P, Chetcuti S, et al. Current role of sodium bicarbonato- based preprocedural hydration for the prevention of contrast induced acute kidney injury: a meta-analysis. Am Herat J 2008; 156: 414-21.

60. Brar S, Hiremath S, Dangas G, et al. Sodium bicarbonate for the prevention of contrast induced kidney injury: a systematic review and meta-analysis. Clin J Am Soc Nephrol 2009; 4: 1584-92.

61. Meier P, Ko D, Tamura A, et al. Sodium bicarbonate based hydration prevents contrast induced nephropathy: a meta-analysis. BMC Med 2009; 7: 23-35.

62. Trivedi H, Nadella R, Szabo A. Hydration with sodium bicarbonate for the prevention of contrast induced nephropathy: a meta-anslysis of randomized controlled trials. Clin Nephrol 2010; 74: 288-96.

63. Kunadian V, Zaman A, Spyridopoulos I, et al. Sodium bicarbonate for the prevention of contrast induced nephropathy: a meta-analysis of published cliniucal trials. Eur J Radiol 2011; 79: 48-55.

64. Kshirsagar A, Poole C, Motti A, et al. N-acetylcysteine for the prevention of radiocontrast induced nephropathy: a meta-analysis of prospective controlled trials. J Am Soc Nephrol 2004; 15: 761-9.

65. Pannu N, Manns B, Lee H, Tonelli M. Systematic review of the impact of $\mathrm{N}$ - acetylcysteine on contrast nephropathy. Kidney Int 2004; 65: 1366-74.

66. Bagshaw S, Ghali WA. Acetylcysteine for the prevention of contrast - induced nephropathy after intravascular angiography: a systematic review and meta-analysis. BMC Med. 2004; 2: 38.

67. Liu R, Nair D, Ix J, et al. N-acetylcysteine for the prevention of contrast induced nephropathy. A systematic review and meta-analysis. J Gen Med 2005; 20: 193-200.

68. Duong M, MacKenzie T, Malenka D. N-acetylcysteine prophilaxis significantly reduces the risk of radiocontrast - induced nephropathy: comprehensive meta-analysis. Catheter Cardiovasc Interv 2005; 64: 471-9.

69. Nallamothu BK, Shojana KG, Saint S. Is Acetylcysteine effectivein preventing contrast related nephropathy? A meta-analysis. Am J Med 2004; 117: 938-47. 